\title{
The Euler Characteristic Is the Unique Locally Determined Numerical Invariant of Finite Simplicial Complexes which Assigns the Same Number to Every Cone*
}

\section{R. Forman}

Department of Mathematics, Rice University, Houston, TX 77251, USA

forman@math.rice.edu

\begin{abstract}
We show that a proof given by Levitt in [L] suffices to prove the stronger theorem stated in the title.
\end{abstract}

Let $\mathcal{S}$ denote the set of finite simplicial complexes, where we consider two complexes to be the same if they are combinatorially equivalent. Say a function

$$
\rho: \mathcal{S} \rightarrow R
$$

is locally determined if there is a function

$$
d: \mathcal{S} \rightarrow R
$$

such that, for all finite simplicial complexes $M$,

$$
\rho(M)=\sum_{\text {vertices } v \text { of } M} d(\operatorname{link}(v))
$$

(where $\operatorname{link}(v)$ denotes the link of $v$ in $M$, and we sometimes write $\operatorname{link}(v, M)$ if there is possible confusion). In this case, we write $\rho=\rho_{d}$. We are primarily interested in those locally determined functions with topological significance. For example, a natural problem is to find all locally defined functions which only depend on the homotopy type of the underlying complex. This problem was posed, and completely answered, by

\footnotetext{
* This research was partially suported by the National Science Foundation and the National Security Agency.
} 
Levitt. In [L] it is proved that

Theorem A [L]. The only locally determined real-valued homotopy invariants of finite simplicial complexes are multiples of the Euler characteristic.

It is easy to see that the Euler characteristic is, in fact, locally determined. Namely, define

$$
e: \mathcal{S} \rightarrow R
$$

by setting, for any finite simplicial complex $N$,

$$
e(N)=1+\sum_{i} \frac{(-1)^{i+1}}{i+2}(\text { number of } i-\operatorname{simplices} \text { in } N) .
$$

Then, for any finite simplicial complex $M$,

$$
\rho_{e}(M)=\chi(M)
$$

where

$$
\left.\chi(M)=\sum_{i}(-1)^{i} \text { (number of } i-\operatorname{simplices~in~} M\right)
$$

is the Euler characteristic of $M$.

Levitt also proves the following refinement of Theorem A.

Theorem B [L]. Let $\rho_{d}$ be any locally determined real-valued invariant of finite simplicial complexes which is a homotopy invariant, then there is a constant $c$ so that $d=c \cdot e$.

Our goal in this paper is simply to point out that an argument used in [L] suffices to give a simple direct proof of the stronger theorem stated in the title. We quickly review some standard definitions. For any simplicial complex $N$, cone $(N)$ is the join of $N$ and a single vertex. We say a simplicial complex $M$ is a cone if $M=\operatorname{cone}(N)$ for some $N$. In the proof of Theorem B in [L], Levitt essentially proves the following theorem.

Theorem 1. Let $\rho_{d}$ be any locally determined real-valued function on the set of finite simplicial complexes. Suppose there is a constant $c$ so that $\rho_{d}(M)=c$ whenever $M$ is a cone. Then $d=c \cdot e$.

Before proving this theorem we note that since all cones are homotopy equivalent, every locally determined homotopy invariant satisfies the hypotheses of Theorem 1 . Thus Theorem 1 implies Theorem B (and hence Theorem A). It is interesting to observe that Theorem 1 implies that the converse is true. Namely, any locally determined invariant which gives the same answer for every cone is a homotopy invariant. Moreover, every cone is collapsible, and hence all cones are simple-homotopy equivalent (see [C] for definitions). Therefore, the above theorem implies the following strengthening of Levitt's Theorem A. 
Corollary 2. The only locally determined real-valued simple-homotopy invariants of finite simplicial complexes are multiples of the Euler characteristic.

We actually prove something somewhat more general. Namely, $\rho_{d}$ need not be defined on all finite simplicial complexes. Say

$$
\mathcal{T} \subset \mathcal{S}
$$

is star-closed if:

(i) $\mathcal{T}$ contains the simplicial complex consisting of a single vertex.

(ii) $\mathcal{T}$ is closed under taking stars. That is, if $M \in \mathcal{T}$ and $v$ is a vertex in $M$, then $\operatorname{star}(v, M) \in \mathcal{T}$.

We will prove the following generalization of Theorem 1 .

Theorem 3. Let $\mathcal{T} \subset \mathcal{S}$ be any star-closed set, and let $\rho_{d}$ be any locally determined real-valued function on $\mathcal{T}$ such that there is a constant $c$ with $\rho_{d}(M)=c$ for every cone $M \in \mathcal{T}$. Then $d=c \cdot e$.

The set of combinatorial manifolds with (possibly empty) boundary is star-closed. Hence we have the corollary

Corollary 4. Let $\rho_{d}$ be any locally determined function on the set of combinatorial manifolds with (possibly empty) boundary such that there is a constant $c$ such that $\rho_{d}(M)=c$ whenever $M$ is a cone, then $d=c \cdot e$.

This implies Theorem $\mathrm{A}^{\prime \prime}$ of $[\mathrm{L}]$, where there is the stronger hypothesis that $\rho_{d}$ be a PL homeomorphism invariant.

Proof of Theorem 3. (The reader should note that we are following the proof of Levitt's Theorem B in [L], and we include this proof merely for the sake of being self-contained.) Let $\rho_{d}$ satisfy the hypotheses of the theorem. Without loss of generality, we may assume that, for every cone $M, \rho_{d}(M)=1$. (Namely, if $c \neq 0$ we can consider $\rho_{d^{\prime}}$ where $d^{\prime}=(1 / c) d$. If $c=0$ we can consider $\rho_{d^{\prime}}$ where $d^{\prime}=d+e$.) Our goal is to show that, for each finite simplicial complex $M \in \mathcal{T}$ and every vertex $v \in M, d(\operatorname{link}(v))=e(\operatorname{link}(v))$. The proof will be by induction on the number $\ell(v)$ (or $\ell(v, M)$ ) of simplices in the link of $v$ in $M$.

Suppose $\ell(v)=0$. Let $M^{\prime}=\operatorname{star}(v)$. Then $M^{\prime}$ consists only of the vertex $v$, and by hypothesis $M^{\prime} \in \mathcal{T}$. Since $\operatorname{star}\left(M^{\prime}\right)$ is a cone (on the empty set), we have

$$
1=\rho_{d}\left(M^{\prime}\right)=d\left(\operatorname{link}\left(v, M^{\prime}\right)\right) .
$$

However, $\operatorname{link}\left(v, M^{\prime}\right)=\emptyset=\operatorname{link}(v, M)$, so $d(\operatorname{link}(v, M))=1$. On the other hand, $1=e(\operatorname{link}(v))$. Thus, if $\ell(v)=0, d(\operatorname{link}(v))=e(\operatorname{link}(v))$.

Suppose $d(\operatorname{link}(v))=e(\operatorname{lin} k(v))$ whenever $v \in M \in \mathcal{T}$ satisfies $\ell(v) \leq k-1$. Let $M \in \mathcal{T}$ and let $v$ be a vertex in $M$ satisfying $\ell(v, M)=k$. Let $M^{\prime}=\operatorname{star}(v, M)$. Then 
$M^{\prime} \in \mathcal{T}$ is a cone, so

$$
1=\rho_{d}\left(M^{\prime}\right)=\sum_{\operatorname{vertices} w \text { in } M^{\prime}} d\left(\operatorname{link}\left(w, M^{\prime}\right)\right) .
$$

It is easy to see that, for each vertex $w$ of $M^{\prime}, \ell\left(w, M^{\prime}\right) \leq k$. Moreover, if $\ell\left(w, M^{\prime}\right)=k$, then $\operatorname{link}\left(w, M^{\prime}\right)$ is combinatorially equivalent to $\operatorname{link}\left(v, M^{\prime}\right)=\operatorname{link}(v, M)$. Define

$$
\begin{aligned}
& V_{1}=\left\{\text { vertices } w \text { in } M^{\prime} \text { with } \ell\left(w, M^{\prime}\right)<k\right\} \\
& V_{2}=\left\{\text { vertices } w \text { in } M^{\prime} \text { with } \ell\left(w, M^{\prime}\right)=k\right\}
\end{aligned}
$$

and let $n=\# V_{2}$. By induction, for all $w \in V_{1}$,

$$
d\left(\operatorname{link}\left(w, M^{\prime}\right)\right)=e\left(\operatorname{link}\left(w, M^{\prime}\right)\right) .
$$

For all $w \in V_{2}$,

$$
d\left(\operatorname{link}\left(w, M^{\prime}\right)\right)=d\left(\operatorname{link}\left(v, M^{\prime}\right)\right)=d(\operatorname{link}(v, M)) .
$$

Substituting into formula (1), we have

$$
1=n \cdot d(\operatorname{link}(v, M))+\sum_{w \in V_{1}} e\left(\operatorname{link}\left(w, M^{\prime}\right)\right) .
$$

On the other hand, we already know

$$
1=\chi\left(M^{\prime}\right)=\sum_{\operatorname{vertices} w \operatorname{in} M^{\prime}} e\left(\operatorname{link}\left(w, M^{\prime}\right)\right)=n \cdot e(\operatorname{link}(v, M))+\sum_{w \in V_{1}} e\left(\operatorname{link}\left(w, M^{\prime}\right)\right) .
$$

Hence we must have

$$
d(\operatorname{link}(v, M))=e(\operatorname{link}(v, M)),
$$

as desired.

\section{References}

[C] M. Cohen, A Course in Simple Homotopy Theory, Graduate Texts in Mathematics, Vol. 10, SpringerVerlag, New York-Berlin, 1973.

[L] N. Levitt, The Euler characteristic is the unique locally determined numerical homotopy invariant of finite complexes, Discrete Comput. Geom. 7 (1992), 59-67.

Received April 2, 1999, and in revised form October 6, 1999. 